\title{
SAXONICA.
}

\section{Das Taufgelöbnis und der Indiculus superstitionum.}

Die frage nach der heimat und entstehungszeit des Sächsischen taufgelöbnisses (in Wadsteins ausgabe der Kleineren as. sprachdenkmäler, nach der ich im folgenden citiere, s. 3) und des in unmittelbarem anschluss an dieses überlieferten sogenannten Indiculus superstitionum et paganiarum (ebenda s. 66) ist noch immer nicht $\mathrm{zu}$ einer befriedigenden lösung geführt worden. Auch die bemerkungen, mit denen der jüngste herausgeber die merkwürdigen denkmäler begleitet hat (s. 119 . 142), bedeuten keinen irgend nennenswerten fortschritt, fassen vielmehr nur die bisherigen aufstellungen in kürze zusammen. Die im folgenden vorgetragene neue auffassung der heimat des Taufgelöbnisses ist kürzlich in einer flüchtig hingeworfenen anmerkung (Zs. fda.43,345) von Wrede zuerst öffentlich ausgesprochen worden: dem gegenüber darf ich bemerken, dass die grundlinien meines aufsatzes bereits concipiert waren, als sein aufsatz über die heimat der as. bibeldichtung in meine hände kam.

Jeder neue versuch, das schwierige problem zu lösen, muss der natur der sache nach mit einer erörterung über die handschrift einsetzen, in der uns die in rede stelsenden stücke überliefert sind. Der Cod. palatinus 577 der Vaticana. aus dem anfang des 9.jh.'s stammend und mit ags. schrift geschrieben, enthält in der hauptsache den ersten teil der. canonensammlung des kleinen Dionysius. Was von einer zweiten hand hinter den canones eingezeichnet ist, geht uns hier nichts an. Dagegen ist, vor ilhnen ein, wie die schlussformel explicit deo yratias beweist, fïr sich abgeschlossener 
toil ringreheftet, der sehr verschiedenartige aufzeichnungen, daruntar auch unsere beiden denkmäler enthält. Die beste iibersicht des inhalts gibt, scherer in den Ienkm. 23, 317 (vgl. auch Wadstein s. 119), mit dessen weiteren aufstellungen über zweck und heimat dieses teilcodex wir uns zunächst ausrinander\%usetzen haben.

Nachdem Scherer bemerkt hat (s. 318), dass die datierbaren stiucke dieser teilhs. chronologisch geordnet sind und diese reihe die jahre 742 und 765 zum anfangs- und endpunkt hat, spricht er (s. 319) die ansicht aus, die ganze teilhs. sei 'offenbar mit beziehung auf die Sachsenmission in Fulda zusammengestellt'; auf seine zeitliche bestimmung dieser zusammenstellung komme ich später zurück. Auffällig bleibe allerdings, dass die wichtige capitulatio Karls de partibus Saxoniae (Boretius, Capitt. reg. Franc. 1, 68) nicht darin enthalten sei; die zusammensetzung des codex, in den sie bei dessen bestimmung zweifellos aufgenommen worden wäre, müisse also wol vor jene capitulatio fallen, was demgemäss auch angenommen wird. Unbedingte zustimmung hiat diese ansicht Scherers nur bei Gallée (As. sprachdenkm. s. 245) gefunden. Gegen die von ihm vertretene ansicht, dass die ganze hs. beziehung zur Sachsenmission habe, und gegen die herleitung derselben aus Fulda sind gewichtige einwände von Jostes (Zs. fda.40, 185) und Koegel (Gesch. d.d. lit. 1, 2, 444; anders noch in Pauls Grundr. ${ }^{1}$, 1, 244) erhoben worden. Der Fuldaer ursprung allein wurde weiterhin angenommen, ohne auf eine analyse der einzelnen teile des codex einzugehen, von Heyne (Klein. and. denkm.2 s.xrrr), Kelle, Gesch. d. d. lit. 1, 44) und Schlüter (bei Dieter, Laut- u. formenl. der agerm. dial. s. xxIx) und erscheint auch Hauck 'nicht unwahrscheinlich' (Kirchengesch. Deutschl. 22, 392, anm. 1); energischen einspruch gegen diese provenienz des codex erhebt von einer ganz andern seite der betrachtung her Falk in seinen wertvollen Untersuchungen über die ehemalige dombibliothek zu Mainz (s. Iv. 11).

Eine musterung der einzelnen bestandteile der hs. zéigt deutlich, dass von einer beziehung zur Sachsenmission nur bei einigen wenigen die rede sein kann (Koegel s. 444). Die beiden ersten stücke De diversis causis, De lapsu episcopi vel presbyteri und Dicta Hicronymi presbyteri sind ganz allgemeinen charakters 
und legen keinerlei specielle beziehungen nahe. Es folgen die beschlüsse der ersten beiden austrasischen synoden Karlmanns von 742 und 743, von denen die erste an einem uns unbekannten orte, die zweite zu Estinnes abgehalten wurde (Boretius 1, 24. 26; vgl. auch MG. Epist. 3, 310). Ueber die kirchengeschichtliche bedeutung dieser synoden orientiert Hauck $\left(1^{2}, 502.514\right)$ : Karlmann hatte sich nichts geringeres als eine gründliche reformation der austrasischen kirche an haupt und gliedern vorgesetzt und verfügte in der ersten synode in die bisherige kirchliche verwirrung tief einschneidende massregeln und vorschriften für die disciplin der geistlichkeit und das religiöse leben der gemeinden; in Estinnes wurden die im vorhergehenden jahre gegebenen verordnungen fast nur bestätigt und in kleinigkeiten ergänzt. Die ideale höhe der hier erstrebten zustände lässt naturgemäss weder an eine rasche durchführung der geplanten reformen noch an eine expansive stärke denken, wie sie eine auf dauernden erfolg hoffende missionstätigkeit zur nctwendigen voraussetzung hat. Von einer solchen auf sächsischem gebiete oder anderswo ist daher auch gar keine rede: zwar beschliesst der fünfte paragraph der ersten synode gegen eine reihe paganiae, darunter auch das dann wider in unserm Indiculus $(66,18)$ erwähnte niedfyr vorzugehen; doch darf man daraus wol nicht $z u$ viel schliessen, denn in Karls erstem capitulare von 769 (Boretius 1,44 ), das eine reihe von paragraphen aus jenem ersten des Karlmann wörtlich herübernimmt (so gering war der erfolg in fast einem menschenalter gewesen; vgl. Hauck $2^{2}, 280$ ), ist gerade diese schon durch einführung der volksmässigen deutschen bezeichnung prägnant hervorstechende stelle wider fortgelassen worden. Die Nomina episcoporum, qui missi sunt a Romana urbe ad mardicandum in Gallia haben widerum mit Sachsen nichts zu tun. Fin gleiches dürfte von dem nun folgenden verzeichnis der 7(i2 oder 763 in Attigny versammelten bischöfe (Boretius 1. 2:21) gelten: alles was wir von dieser synode wissen (ich folge hitr wider Hauck $\left.2^{2}, 67\right)$, ist nur, dass sich ihre toilnelhmer anf anregung Chrodegangs von Metz zum abschluss rines guloctsvereins entschlossen, also eime tatsache von rein gemütlicher bedeutung. Hinter 'T'aufgelïbnis mol Indiculus. dic an diexer

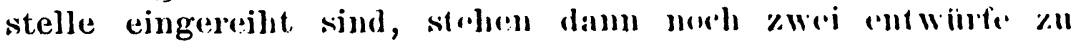


prodiglen (dass sie das sind und nicht, wie man früher meinte, bischöfliche ansprachen, hat Hallck $2^{2}, 398$, anm. 2 dargetan), bei denen die beziehung auf die Sachsenmission möglich ist, abcr auch nicht mehr: die von Hauck fiir diese beziehung geltend gemachten gı unde sind zwar bestechend, gewinnen aber auch für ilın erst durch die 'wahrscheinliche bestimmung der lıs.' volle beweiskraft, die ihnen also, wenn man meinen bisherigen erörterungen beistimmt, wider entzogen sein würde. Warum sollte an sich nicht auch an Friesenmission oder vielleicht, wozu allerdings der gesammtcharakter der stücke mir nicht recht $\mathrm{zu}$ passen scheint, an musterentwürfe ohne bestimmte locale beziehung gedacht werden können? Abgesehen ron Taufgelöbnis und Indiculus bleibt also nur für diese predigten eine nicht gar zu illusorische möglichkeit, dass sie für die Sachsenmission bestimmt sein könnten.

Noch müssen wir mit zwei worten auf die von Scherer hervorgehobene tatsache eingehen, dass die so eminent wichtige Capitulatio de partibus Saxoniae nicht unserm codex einverleibt worden ist. Was ihre datierung anbetrifft, die Boretius zu allgemein innerhalb der jahre 775-790 ansetzte, so nehme ich mit Hauck $\left(2^{2}, 386\right.$, anm. 1) an, dass sie 787 oder 788 erlassen wurde, also zweifellos vorhanden war, als unser codex geschrieben wurde. Es gibt keinen kaiserlichen erlass die christianisierung und sittliche cultivierung Sachsens betreffend (die politische oder staatswirtschaftliche bedenklichkeit mancher vorschriften, die sich im laufe der späteren entwicklung der dinge bitter gerächt haben, habe ich hier nicht zu erörtern), der sich an fundamentaler bedeutung mit dieser Capitulatio nur irgend vergleichen liesse. Ihr fehlen in einer hs. die eigens für sächsische verhältnisse zusammengestellt sein soll, muss aufs höchste wunder nehmen. Scherer (s. 319) sah sich deshalb genötigt, die zusammensetzung des codex vor den erlass der Capitulatio zurückzuverlegen: wir haben jedoch durchaus keinen grund anzunehmen, dass unsere hs. älter ist als das beginnende 9. jh. Jostes (s. 186) sagt mit recht, dass, die Capitulatio, wenn sie wirklich in der hauptvorlage unseres codex nicht stand, leicht anderswoher hätte entnommen werden können und müssen, wenn die hs. wirklich zu dem angenommenen zwecke zusammengestellt wurde, und möchte aus dem 
fehlen der Capitulatio 'eher umgekehrt schliessen, dass die hs. gar nicht für die Sachsenmission bestimmt war'. Diesem schlusse wird man in anbetracht der bisherigen darlegungen, wie ich glaube, beistimmen müssen. Bedenkt man ferner, dass das Taufgelöbnis in seinem zweiten teile, der confessio, unvollständig ist (Kelle s. 43), sowie in seinem ersten, der abrenuntiatio, abweichungen in der anordnung der fragen und der formulierung der antworten von dem vorgeschriebenen lateinischen texte darbietet (Scherer s. 316), in beiden fällen also keinen irgend officiellen text aufweist (vgl. auch Wilmanns' bemerkungen in den Gött. gel. anz. 1893, s. 538, der deshalb aufzeichnung aus dem gedächtnis annimmt, was mir nicht glaublich ist und auch zur erklärung nicht genügen würde), so dürften wir wol zu dem endresultate gelangen: Scherers these, dass unsere ganze hs. mit beziehung auf die Sachsenmission gewissermassen kanonisch zusammengestellt worden sei, ist als unerweislich abzulehnen.

Wie steht es dann weiter mit der von Scherer behaupteten provenienz des codex aus Fulda? Die ansichten jüngerer forscher habe ich oben ïbersichtlich zusammengestellt. T'nser codex enthält wie eine ganze reihe anderer (vgl. darüber jetzt Falk s. 2.25) den vermerk, dass er der bibliothek des Martinsdomes zu Mainz angehört hat. Dieser wurde im jahre 1036 durch erzbischof Bardo geweiht und übernahm kleriker und inventar von dem älteren Mariendome, dessen gründung, nicht mit voller genauigkeit bestimmbar, etwa in die mitte des 8. jh.'s $\mathrm{zu}$ setzen ist (Hauck $2^{2}, 796$ ). So wanderte denn auch der büchervorrat des alten domes um 1036 nach dem neuen hinüber (Falk s. 11). Im laufe des mittelalters ist dann, wie Jostes gezeigt hat (s. 129; vgl. auch Falk s. 116. 124. 1:33), weiter auch die bibliothek des klosters St. Alban (gegründet 8(1)5; vgl. Hauck 22, 567) der dombibliothek einverleibt worden. 1)a keine einzige der alten Mainzer hss. eine andere provenienz. aus Fulda oder sonstwoher, bekundet, so hat .Jost.es mol ihm folgend Koegel mit, recht betont, dass zu der annahme. unser codex stamme aus Fulda, mindestens keine noitigung vorlingt. und es vorsichtiger ist bei Mainz stehen zu bleiben. Anch lialk hat jüngst (s. Iv) die für liulda geltend gemachton grimde als

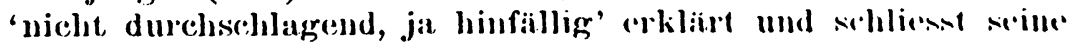


rrirterung ïber die frage (s. 12) mit dem satze: 'es müsste als rin manz tigenes verhängnis bezeichnet werden, wenn alle altun buchlackel mit dem provenienzvermerk Fulda zu verlust reratch wiiren'. Scherers annahme beruhte wesentlich auf eincr ailteren noti\% Giescbrechts, ler eine reihe Mainzer hss. auf mruml der darin gebrauchten ags. schrift und einer durchaus nicht gesicherten beziehung zu Marianus Scotus dem berühmten Fuldier scriptorium zuweisen wollte. Inzwischen hat uns Kauffmamn in seinen bedeutungsvollen studien über ahd. orthoglaphie (Germ. 37, 243) gelehrt, dass die kenntnis der ags. schrift aller wahrscheinlichkeit nach in allen literarisch regsamen klöstern verbreitet gewesen ist und neben Fulda gerade in Mainz besonders im schwange war (vgl. ebenda s.258 und Philol. stud. für Sievers s. 125). Die existenz eines oder gar mehrerer Mainzer scriptorien (vgl. Falk s. 3) kann keinem zweifel unterliegen, wenn man nicht etwa annehmen will, die metropole der deutschen kirche habe ihren literarischen bedarf ständig von ausserhalb beziehen müssen. Dass ein reges literarisches leben in Mainz im ausgehenden 8. und beginnenden 9. jh. herschte, ist durch die geistige bedeutung der drei erzbischöfe Lul, Rikulf und Heistolf ohne weiteres klar (vgl. über sie Hauck 22, 46. 151, anm. 3). Alle jene hss. können demnach sehr wol in Mainz selbst, sei es für den alten Mariendom, sei es für das kloster St. Alban, geschrieben sein, ja es spricht alle wahrscheinlichkeit dafür, dass dies der fall war. Es liegt also kein grund vor, mit Scherer den ursprung unserer hs. ausserlialb Mainz, speciell in Fulda, zu suchen.

Von dem bisher festgelegten fundamente aus können wir nun den an das Taufgelöbnis im besondern sich anknüpfenden fragen und problemen nähertreten. Es wird sich empfehlen, zunächst die sogenannte interpolation in der dritten antwort der abrenuntiatio zu besprechen. Die überlieferung dieser antwort ist nach der bis heute allgemein giltigen auffassung folgende (3,9): end ec forsacho allum dioboles uercum and wordum, Thunaer ende Wôden ende Saxnôte ende allum them unholdum, the hira genotas sint. Alles was nach wercum steht, erklärte Scherer (Denkm. 23,316 und, nach Holtzmanns ohne 
grunde ausgesprochenem widerspruch Germ. 9, 74, ausführlicher in den Klein. sclir. 1, 577) für einen späteren zusatz, eine interpolation. Seine begründung lautet (s. 316): 'die annahme einer interpolation ergibt sich daraus, dass die eingeklammerten worte in der frage fehlen, dass für die worte Thunaer u.s.w. die einzig passende stelle nach der ersten frage gewesen wäre und dass für denselben begriff, der vorher durch diobol, diabol gegeben ist, hier wie im Fränkischen taufgelöbnis, dem grossen Bibelglossar und den Hymnen ... unholda gebraucht wird'. In dem letzten grunde sieht er (s. 577) eine 'äussere bestätigung', die jene beiden erwägungen $z u$ stützen geeignet sei. Weiter heisst es bei ihm (s. 577): 'man glaubte jene einfachen drei fragen nicht genügend; es schien notwendig, die heidnische poesie (die teufelsworte) ausdrücklich mit einzuschliessen und dem begriff des teufels durch die nennung der hauptgötter die vollste und unzweideutigste bestimmtheit zu verleihen. Die hervorgehobenen worte, an den rand eines exemplars der ursprünglichen einfacheren formeln geschrieben, waren nichts als eine andeutung und erinnerung für den taufenden, auf welche gegenstände sich seine abschwörungsfragen sonst noch zu erstrecken hätten, und es blieb ihm selbst überlassen, die fragen zu formulieren.' Diese auffassung Scherers fand bedingungslose zustimmung bei Heyne ( 2 s. 88), Kelle (s. 43) und Gallée (s. 246); die ebenso unbedingte ablehnung von Jostes (s. 189) berücksichtigt nur Scherers ersten punkt und scheint mir auch für diesen bedeutungslos zu sein. Eine wichtige und die ganze frage erheblich klärende modification an dem wortlaut Scherers nahm Hauck $\left(2^{2}, 392\right.$, anm. 1) vor, indem er die wahl des ausdrucks 'interpolation' beanstandete, 'da er den schein erweckt, als sei der den gewöhnlichen tauffragen beigefügte zusatz nur geschrieben, nicht gebraucht, während es sich offenbar $1 \mathrm{~m}$ eine aus praktischen erwägungen hervorgegangene beifügung lıandelt'. Dieselbe ansicht sprach, olme Hauck zu citieren. bald darauf Wilmanns (Gött. gel. anz. 189:3, s. 5:38) aus: auch (י) plemisiert gegen den ausdruck 'interpolation', hiilt jedoch daran fest, dass die betreffenden worte ein zusatz sind, der min vorliegende text also auf einer contamination beruht, die mit hilfe derselben alten formel $\mathrm{zu}$ stande kam, dice ms in dor dritlall frage des Friankischen taufgelöbnisses (1)(1nkm. 5.2. 5; allırdings 
rntspricht, diese dritte frage der süchsischen zweiten, was Wilmanns nicht horvorhebt) iiberliefert ist, 'nur dass, was sie in alleremeinen ausdrü̈cken verlangt, hier den verhältnissen des silchsenvolkes gemïss specialisiert ist'. Koegel endlich (dem friilur' in l'auls ('rruudr. 2', 1, 244 die annalıme eines zusatzes 'durchaus unwahrscheinlich' vorgekounmen war) hat (s. 448) fïr die so modificierte ansicht Scherers die treffende formulierung gefunden, die sogenannte interpolation könne wol 'ein mit dem ïbrigen gleich alter eventualzusatz sein, den man anwendete, wo besondere gründe dazu vorlagen'. Soviel über den heutigen stand der ansichten.

Mit vollem recht hat Scherer (s. 577) mit rücksicht auf Holtzmanns opposition seine oben citierten schlüsse als 'einfach, natürlich und selbstverständlich' charakterisiert: man wird ihnen, glaube ich, durchaus beistimmen müssen. Andrerseits aber ist ebenso klar, dass dies nur mit einer modification in der ron Hauck, Wilmanns und Koegel übereinstimmend eingeschlagenen richtung geschehen kann. Dass aus dem nebeneinander von diobol und unholda eine verschiedenheit des sprachgebrauchs für text und zusatz $\mathrm{zu}$ erschliessen sei, ist von Kauffmann (Beitr. 18, 153) mit guten gründen in abrede gestellt worden: es sei durchaus nicht derselbe begriff in dem einen falle mit diobol, in dem andern mit dem plural von unholda bezeichnet worden; das lehre schon der gebrauch des plurals. Dass diobol wirklich eine specifisch ags. bezeichnung ist und ihr vorkommen hier, wie Kauffmann will, auf ein eindringen ags. terminologie in die deutsche kirche zurückgeführt werden muss, scheint mir nicht so sicher erwiesen (vgl. Koegel s. 446): wir wissen von den anfängen der deutschen kirche in dieser hinsicht doch zu wenig und das zufällig erhaltene spärliche sprachmaterial reicht für einen solchen schluss meines erachtens nicht hin. Das Fränkische taufgelöbnis beweist uns, wie Wilmanns gesehen hat, dass vor der, vielleicht sogar noch neben der lakonischen formel des ordo romanus specialisiertere, den individuellen verhältnissen und localen bedürfnissen / angepasste ausführlichere formeln in geltung waren. So wird auch in unserm denkmal dem farblosen, so zu sagen officiellen terminus, der noch dazu ein fremdwort ist, durch die nennung der gütternamen und das ihnen beigefügte einheimische 
unholdum ${ }^{1}$ ) individuelles leben verliehen. Wir haben ein interessantes capitulare Karls vom jahre 811 (Boretius 1, 162), eine art programm für eventuelle synodalverhandlungen und besprechungen mit den kirchenhäuptern, in welchem $(\S 9)$ ein tieferes inneres verständnis der lakonischen tauf- und abschwörungsformeln und ihrer termini von den clerikern verlangt wird, die bestimmt sind, durch leben und lehre den laien ein vorbild zu geben. Wenn es nun hier heisst, dass selbst die cleriker gut daran tun, sich einmal die frage ernstlich zu überlegen, quis sit ille satanas sive adversarius, cujus opera vel pompam in baptismo renuntiavimus, wie viel mehr musste es einem denkenden missionar nötig erscheinen, seinen täuflingen klarere vorstellungen vom wesen und den werken des diabolus beizubringen. Wenn dies nun auch sicherlich in erster linie in der predigt geschah, so haben wir in solchen erwägungen, meine ich, doch auch den psychologischen grund für die eventualzusätze zu den formeln zu suchen, und es wurden wol auch schon früher als 811 derlei kaiserliche fragen der missionierenden geistlichkeit nahegebracht.

Es fragt sich nun, ob man sich bei den bis zu diesem punkte gewonnenen resultaten beruhigen kann, ob alle anstösse beseitigt, alle schwierigkeiten gelöst sind. Ich glaube nicht. Unser eventualzusatz zeigt einen Januskopf: die erwähnung der worte des teufels neben seinen werken lässt ihn als zur dritten (nach dem ordo romanus eigentlich zweiten), die nennung der götternamen, wie schon Scherer bemerkt hat, als zur ersten frage gehörig erscheinen. Beides zugleich ist nicht möglich, jedes einzelne schief und unlogisch: es muss hier etwas nicht in ordnung sein, und zwar steckt meiner überzeugung nach der fehler in den ersten beiden worten des zusatzes. Wie der schreiber unseres denkmals an einer andern stelle zwei worte seiner vorlage falsch getrennt hat $(3,10 \mathrm{cn}$ dewoden statt des richtigen ende Wôden) und zwar ebenfalls innerhalb des zusatzes, so hat er hier ein zusammengehöriges einheitliches wort in zwei auseinandergerissen. Statt and

') Ob man mit Scherer (s. 578) und Korgel (s. 448) (ein fomininum nach desn ahd. oder mit Kauffmann (s. 154), dem neuerdings Wadstein (s. 2:35) folgt, ein masculinum nach dem ags. ansetzen will, ist. dinc fruge von untergeordneter bedeutung. 
"omblum ist andur)rlum zu lesen und diese form ist nichts anderess als der dat. pl. von cueclecordi: da das wort im as. sonst nII im mom. oler ace. sg. belegt ist (Hel. 930. 1759. 2432.4040. 4(155. 40)1. 5!)(j7. (ien. 176. 206. 2\%9; in den kleineren denkmiilern fehlt es), so ist nicht auszunachen, ob die zu erwartende endung -ium durch lautliche entwicklung (vgl. Holthausen, As. clementarb. $\$ 17: 3$ und Schlüter bei Dieter 1, 279) oder etwa durch angleichung an wordum ihr stammhaftes $i$ verloren hat. I)er sinn des wortes, hinter dem nach moderner interpunction ein kolon zu denken wäre, ergibt sich, wenn wir beachten, lass in unserm denkmal jede antwort durch den lateinischen vorsatz resp. eingeleitet wird, den man seit Boretius $(1,222)$ und Gallée (s. 248), ich sehe nicht ein. warum, immer in respondeat auflöst (so auch Wadstein 3, 5. 7.9). Liest man responso oder responsis, so ist anduordum davon die wörtliche übersetzung. Der ganze satz mit den götternamen sollte in der antwort gebraucht werden und gehört, da nun nicht mehr von worten des teufels die rede ist, klärlich zur ersten frage, wie schon Scherer sah: an stelle des oder neben den farblosen diabolus traten die germanischen hauptgötter der gegend, für die die formel bestimmt war, sammt den ihnen gleichwertigen unholdum. Unser eventualzusatz stand also wahrscheinlich, wie schon Scherer annahm, in der vorlage unseres codex am rande neben der formel für die abrenuntiatio und solltezur ersten frage in beziehung stehen, wurde aber vom abschreiber falsch verstanden und der dritten frage angehängt.

Bei dieser auffassung von and wordum als andwordum ist nicht nur sachlich alles in ordnung, sondern es fallen damit zugleich drei steine des anstosses weg, die bei der bisherigen ansicht schwierigkeiten bereiteten. Erstens die merkwürdige übersetzung von opera des teufels durch 'werke und worte', wobei nicht klar ist, was man sich eigentlich unter den worten des teufels zu denken hat: sie müssten geradezu ohne besonderen sinn rein der alliterierenden formel zu liebe hinzugefügt worden sein, denn mit Scherer darunter die heidnische poesie zu verstehen, wird ohne parallelstellen kaum angehen; wie käme auch eine rücksichtnahme auf diese in eine kirchliche formel? Zweitens ist der wortlaut dieser alliterierenden formel 
höchst auffällig, worauf Koegel (s. 447) hingewiesen hat: die formel heisst sonst stets vordum endi werkum, also gerade umgekehrt (reiche as. und ags. belege in Sievers' Heliand s. 466 und anm. 1; vgl. ferner Grimm, Rechtsaltert. 14, 10 und Hoffmann, Reimform. im westgerm. s. 60). Drittens verschwindet die form and damit aus dem texte, an die mancherlei behauptungen und schlüsse angeknüpft worden sind. Scherer (s. 316) sah darin einen sicheren anglosaxonismus, und zwar (s. 578) nicht wegen des stammvocals $a$, der auch sonst auf sächsischem boden vorkomme, sondern wegen der weder as. noch ahd. nachweisbaren apokope des schlussvocals vor folgendem consonanten (ähnlich Kauffmann, Beitr. 18, 153). Dem gegenüber wies Koegel (s. 445) mit recht auf ant und end im Keronischen glossar und vor allem auf das ganz gewöhnliche afries. and hin (vgl. Richthofen, Afries. wörterb. s. 604); eher könnte man daher von einem frisonismus reden. Aber ganz abgesehen von diesen auswärtigen parallelen muss in einem texte, der unbetonte silben und formwörter so consequent und einheitlich schreibt (man beachte die 4 for-, die $7 g e$-, die $6 e c$, die 6 -o der 1. sg. praes., die 4 -an im acc. sg. des adjectivs, endlich die 9 -um in den dativen), vereinzeltes and neben consequentem siebenmaligem end, cnde verdacht erwecken. Die hebung dieser drei schwierigkeiten dürfte als ein weiteres moment für dievon mir vorgeschlagene emendation sprechen

Die letzten erörterungen haben uns noch zu einem weiteren nicht unwichtigen ergebnis verholfen: das uns erhaltene Taufgelöbnis ist keine originalniederschrift, sondern eine copie aus einer vorlage. Denn nur durch ein misverständnis der vorlage waren wortlaut und stellung des eventualzusatzes zu erklären. Nach Koegel (s. 448) hätten wir eine erste niederschrift 'nach dem gehör, also aus dem gedächtnisse oder auf grund eines dictates' vor uns: die sandhierscheinungen aber, die er als beweis anführt, kömmen das allein nicht begründen, zumal dann nicht, wenn gewichtigere beobachtungen $z u$ einer anderen auffassung nötigen. liockel selbst spricht sonderbarerweise wenige blätter vorher (s. 111) von einer "vorlage, die dieser copist vor sich hatte". Willoher art diese vorlage gewesen sein mag, dariaber ist natrirlich nichts sicheres zu sagen; hier sind wir anf vermutungen an- 
mewiesen. Irh mörhle: kaum glauben, dass die ganze teilhs., wio sie obru allalysic!t worlen ist, so wie sie vorliegt, ganz IInI mar ans cince vorlage coplert ist, nelime vielmehr an, dass div stïrke nath und nach aus uns nicht mehr erkennbaren motiven zusimmengestellt und verschiedenen vorlagen entnommen wurden. Der niederschrift des Taufgelöbnisses kann, schon wegen seiner unvollständigkeit und unordnung (vgl. oben $\therefore .571$ ) und wegen des vorhandenseins einer randbemerkung, gauz wol ein fragmentarischer entwurf oder eine kladde mit correcturen, schwerlich eine officielle reinschrift $\mathrm{zu}$ grunde liegen. Aber die wunderliche dialektmischung möchte ich schwerlich schon dieser kladde, sondern eher dem copisten zuschreiben.

Damit treten wir der frage nach dem dialekt des denkmals näher oder mit anderen worten der frage, für welche gegend des sächsischen landes es in Mainz aufgezeichnet wurde, in welcher gegend es seinem sprachcharakter nach praktische verwendung finden sollte. Passend hat man sie in erster linie mit hilfe der kirchengeschichte lösen wollen, indem man von dem heimatsorte der hs. und seinen missionsgeschichtlich nachweisbaren oder wahrscheinlichen beziehungen ausgieng. Scherer, der den codex aus Fulda ableiten wollte, hat demgemäss (s. 318) den bezirk der Diemel, der zunächst jenseit der Sachsengrenze an Hessen stösst, also den bereich des späteren bistums Paderborn in übereinstimmung mit Rettberg, der die Fuldaer mission hierher verlegte (vgl. aber neuerdings Hauck 22, 377, anm. 4), als heimat des denkmals, das demmach engrischen dialekt tragen würde (s. 319), bezeichnet; Heyne (2 s. xıII) und Gallée (s. 246) sind ihm darin gefolgt. Dagegen machte Jostes (s. 185) geltend, dass vor der gründung des Paderborner bistums diese ganze gegend nicht zum Mainzer, sondern zum Würzburger sprengel gehörte; Koegels zweifel daran (s. 444) sind durchaus unberechtigt, da wir in der Translatio Liborii ein sicheres historisches zeugnis dafür besitzen (Hauck 22, 377). Uebrigens kommt für beide gelehrte die ganze frage der Fuldaer mission gar nicht mehr in betracht, da sie den codex in Mainz localisieren; trotzdem haben sie eine umschau von diesem ihrem neugewonnenen ausgangspunkt aus $\mathrm{zu}$ halten und damit einen neuen localisierungsversuch $\mathrm{zu}$ machen unterlassen.

Es ist $\mathbf{z u}$ 
fragen, was die beglaubigten kirchengeschichtlichen zeugnisse hierfür ausgeben. Nach Haucks darlegungen $(22,375)$ wurden auf der Paderborner synode von 777 die kirchlichen verhältnisse Sachsens, soweit es überhaupt damals schon der fränkischen machtsphäre unterstand, nicht sowol durch abgrenzung genau bestimmter sprengel als vielmehr durch bildung von einzelnen missionsgebieten geordnet, welche den bestehenden kirchlichen instituten des reiches zur geistlichen versorgung zugewiesen wurden; später sind aus diesen teilgebieten die eigenen sächsischen bistumssprengel erwachsen. Die zuteilung geschah in der weise, dass neben entfernteren bistümern wie Würzburg und Lüttich, vielleicht sogar Reims und Châlons sur Marne, in erster linie die grenzbistümer Utrecht, Köln und Mainz für die ilınen besonders nahe liegenden gegenden herangezogen wurden. So fielen Mainz die südlichen gaue der Engern, soweit sie nicht wie die Paderborner gegend anderweitig vergeben waren, und das benachbarte Ostfalen zu. Was speciell den sächsischen ost- und südostwinkel anlangt, so sind dortige gaue für zwei Mainzische klöster mit allergrösster wahrscheinlichkeit als wirkungsgebiete ihrer mission zu erschliessen, für St. Alban in Mainz und für Hersfeld. Eine verpflichtung Hersfelds zur missionstätigkeit im Hassagau und Friesenfeld erhellt daraus, dass die zehnten dieser gebiete dem dortigen kloster urkundlich zugestanden wurden (vgl. Hauck $2^{2}, 378$, anm. 2 und Wrede, Zs. fda. 43,348; das verzeichnis der zehntpflichtigen orte hat zuletzt Schröder in den Mitteil.d. inst. f. österr. geschichtsf. 18, 1 behandelt); auch Quedlinburg im benachbarten Hardagau stand zu Hersfeld in beziehungen. Für den nördlich angrenzenden gau Nordthüringen wissen wir, speciell für die gegend von Magdeburg, durch . Tostes' glänzende entdeckung eines Magdeburger kalenders in der altsächsischen Genesishs. (vgl. über sie jetzt auch Falk s. 132), dass dort das Mainzer St. Albanskloster tätig war (Hauck 22, 376, anm. : stimmt Jostes bei). Wir sahen oben (s.572), dass unser codex so gut der bibliothek des alten Mainzer Mariendoms wir der des St. Albansklosters entstammen kann; andrerscits wiirda auch möglich sein, dass er für Hersfolder missiomare in Mainz geschrieben wïle; sicherheit ist hire wol sehwerlich \%u reichen, wemn sie nicht der noch immer ansitehende æweite 
band des officiollen katalogs der vaticanischen Palatini viellricht hringt. Vou diesen kirchengeschichtlichen er-

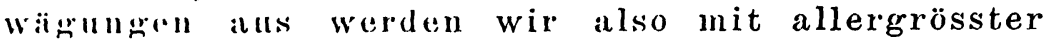
Walischeinlichleit annehmen dürfen, dass unser 'Pauldelähis zur verwendung in einem der ostfälisiluen grate, also in dem reichlich mit ingwäonischen clomenten durchsetzten sprachgebiet Südostsachsens lestiumt war.

Wie verhalten sich zu dieser annahme die sprachformen des denkmals selbst? Bei dem geringen umfang desselben, der durch die vielen wortwiderholungen noch mehr zusammenschrumpft, könmen wir a priori schon kaum auf ein vielgestaltiges beweismaterial uns hoffnung machen: wir werden vielmehr mit wenigem zufrieden sein oder gar mit der tatsache, dass nichts gegen die vermutete localisierung spricht, uns bescheiden muissen. Der wunderliche mischdialekt, in dem uns das denkmal entgegentritt, hat der wissenschaft viel kopfzerbrechen verursacht: noch Jostes nennt ihn in offenbarer ratlosigkeit (s. 190) 'ganz bastardhaftig'; Koegel gewann wenigstens einen schritt boden, indem er Westfalen entschieden ablehnte (s. 446); der jüngste darsteller der altsächsischen grammatik, Holthausen, gelangt gar zu einer vollständigen negation, indem er sowol in seinem Elementarbuch das denkmal im verzeichnis der quellen des altsächsischen (§ 19) ganz fortlïsst, als anderswo (Anz. fda. 26, 35, anm. 1) kategorisch erklärt: ' $z u$ diesen gehört das wunderliche 'Taufgelöbnis gewis nicht!'. 'Trotzdem muss es versucht werden, dies wunderliche sprachgewand noch einmal unter die lupe zu nehmen. Da das scheinbar ags. and durch unsere emendation beseitigt ist, so bleiben von den drei verschiedenen gruppen von spracheigenheiten, die Koegel (s.445) in dem denkmal unterscheidet, nur zwei übrig, die sächsischen und die hochdeutschen elemente.

Betrachten wir zunächst die ersteren, so finden wir allerding's ein sicheres anglofriesisches oder anders ausgedrückt ingwäonisches characteristicum, das aufs beste zu unserer annahme stimmt. Zweimal gleichlautend $(3,18.19)$ ist hâlogan gâst mit $\hat{a}$ aus germ. ai überliefert (ich setze, wie bisher allgemein geschehen ist, hâlogan mit $\hat{a}$ an, da ich mit Koegels a IF. 3, 287 mich nicht $\mathrm{zu}$ befreunden vermag). Dasselbe $\hat{a}$ 
ist fünfmal im Cott., einmal im Mon. des Heliand, einmal in der Genesis belegt (die stellen verzeichnet Schlüter bei Dieter s. 96). Gewöhnlich sieht man darin, wie neuerdings auch Bremer (Pauls Grundr. 32, 862), spuren ags. schreiber; ich glaube jedoch nicht, dass alle diese fälle einheitlich zu beurteilen sind. Für den schreiber des Cott. mag diese erklärung zutreffen, denn formen wie drihtnes, scealt, stcorra, on, thon kann man wol nur einem Angelsachsen zutranen (vgl. Sievers s. xv); derselbe verfiel dann wol auch vereinzelt in sein heimisches scân, ârâs, hâlag. Ueber das einzelne hâlagon im Mon. will ich kein urteil äussern. Wenn wir aber durch Jostes wissen, dass der schreiber der Genesis ein Ostfale war, so werden wir in dem einen lâro neben den zwei lêra doch wol hier auch ein ausgleiten des schreibenden in die heimische ostfälische mundart annehmen können; der schreiber braucht darum so wenig ein Angelsachse gewesen zu sein wie der des Taufgelöbnisses. Man unterscheidet, wie mir scheint, nicht immer streng genug zwischen ags. schrift und ags. lantgebung: jener konnte sich auch ein Deutscher recht gut bedienen, olne notwendig in diese verfallen zu müssen; dieser konnte nachgeben, wem sie angestammt war, auch ohne jene anzuwenden; beides braucht sich nicht gegenseitig zu bedingen, am wenigsten die schrift den laut. Koegel (s. 446) und ihm beistimmend Braune (Bruchst. d. as. bibeld. s. 12) fassen jenes $\hat{a}$ nicht melu als anglosaxonismus, sondern als frisonismus auf; wir vereinigen gewissermassen beides, wenn wir es, wo nicht wie beim cott. ein ags. schreiber auch sonst sich klar zu erkennen gibt, für ingwäonisch halten, falls nichts anderes dieser annahme entgegensteht. In welchen localen grenzen diese ingwäonisch gefärbte mundart gelebt hat, ist eine zur zeit noch nich1 hefriedigend gelöste frage. So bestechend auch Bremers hypothese zweier social getrennter sprachschichten im Sachsenlande (Pauls Grundr. 32, 866) mir gewis wie andern erscheint. N) braucht man doch, auch wenn man ihr im grossen und gan\%en zustimmt, nebenher nicht in abrede zn stellen, dass fiir gr.wisso gebiete auch Jocale umgrenzung angenommen werdell k:III odey muss, wie schon die gaunamen nahelegen. Dass der lu. weis für den süchsischen siidosten erbracht ist. kanll $\mathrm{k}: \mathrm{mm}$

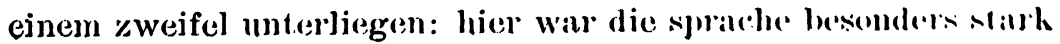




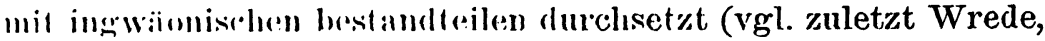
\%. fila. 1:3,:1(1)). lah hemerke, dass ich deshalb so wenig wie

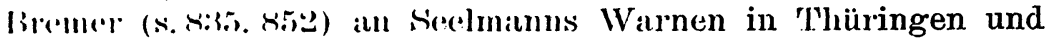
liruler an drer llavel grlaube. - Weiterhin hält Koegel (s. 447) las .r in dron güitternamen suxuôt $(3,11)$ für einen frisonismus, dal silnt hs auf lem sächsischen sprachgebiete allgemein zu ss assimiliert Werde. l)ass der buchstabe $x$ auf ags. schreibmWwhmheit beruht, ist klal; die lautverbindung jedoch, die er hrzeirhnte, war wol zur zeit unseres denkmals noch nicht asimiliert; die chronologie dieses lautïberganges ist ganz IIIxicher (vgl. Holthausen $\$ 215$ und Schlüter bei Dieter s. 281). Auch im südöstlichen Sachsen wird natürlich hs lebendig gewesen sein und wir können auch hier den frisonismus entbehren. I $(\cdot h$ schliesse bei gelegenheit des namens Saxnot hier wltikh eine mythologische bemerkung an. Bekanntlich begegnet ler name ausser in unserm denkmal nur noch in der ags. wenealogie von Essex als Saxnéat (Grimm, Mythol. 34, 379. 382). I)as stimmt vortrefflich zusammen, denn auch unser Saxnot wäre, wenn wir richtig localisieren, ein von Ingwäonen verehrter gott gewesen. Schon Jacob Grimm hat ihn (Klein. schr. 5, 30) mit Freyr-Ing, dem stammgotte der Ingwäonen, identificiert, dessen schwert sjálft vegesli vip jotıa átt (Skírnesmịl 8,3), diese gleichung aber später (Myth.` s. 169. 178) wider fallen lassen zu gunsten des Ziu, dessen ursprüngliche wesensgleichheit mit Freyr er noch nicht erkannte (vgl. Mogk in Pauls Grundr. 3\%, 316. 319). Jedenfalls haben wir kein recht zu der annahme, dass Saxnot ein im ganzen sächsischen gebiete geläufiger göttername gewesen sei. - Die übrigen von Koegel (s. 446) angeführten frisonismen wollen nichts besagen: statt des unumgelauteten forsachistu ist mit Wadstein $(3,4)$ forsaichistu mit umlaut zu lesen (vgl. auch alanehtigan 3,14. 15 und Braune, Ahd. gramm. ${ }^{2} \S 26$, anm. 4); das vorgeschobene $h$ in hira $(3,12)$, mag es nun rein graphisch oder nach analogie des nom. $l \hat{c}$ angetreten sein (Holthausen $\S 332,2$ ), steht nicht nur vereinzelt in der Genesis und im Cott. des Heliand, sondern auch im mfrk. Trierer capitulare (Denkm.66, 9. 12. 13. 19). - Noch sei des merkwürdigen ec (3, 5. 7. 9. 15. 17. 19) mit seinem consequent widerholten $e$ gedacht: es findet sich nur in diesem denkmal; ein zweiter beleg in den Essener 
glossen, den Gallée (s. 47) brachte, ist durch Wadsteins collation der hs. illusorisch geworden (vgl. dessen ausgabe s. 56, anm. 9); daher ist auch die von Koegel (s. 446; doch vgl. s. 652) behauptete beziehung des Taufgelöbnisses zu diesen glossen besser beiseite zu lassen. Dieses $e c$ direct mit dem in heutigen nd. mundarten $z$ wischen Oberweser und Mittelelbe geltenden ele zu identificieren, das schon im mnd. sich aus unbetontem itk neu entwickelt hat, ist schon dieser seiner wahrscheinlichen genesis wegen unmöglich (vgl. auch Tümpel, Nd. stud. s. 75. 132). Es bleibt also nichts anderes übrig, als in diesem südostsächsischen $c c$ eine altertümlichkeit $\mathrm{zu}$ sehen und es als eine directe fortsetzung der fürs urgerm. vorauszusetzenden orthotonierten form *ek (vgl. Streitberg, Urgerm. gramm. s. 51) zu fassen.

Ueber die hochdeutschen elemente in der überlieferung unseres denkmals bleibt nach Koegel (s. 445) wenig zu sagen. Er führt als solche an: forsacho, forsaichistu (3, 4. 5. 7. 9), allêm $(3,11$; durch correctur in as. allum verwandelt) und got, gotes $(3,14.15 .17)$. Uebersehen ist sint $(3,12)$ für as. sind. Diese hochdeutschen elemente fallen sicher dem Mainzer abschreiber zur last, der demnach ein Nichtsachse gewesen sein dürfte. Trotz dieser vereinzelten fehler hat er doch im ganzen, worauf ich schon oben (s. 577) mit einzelbeispielen hingewiesen habe, recht consequent und gewissenhaft copiert. Von seiner vorlage nehme ich deshalb an, dass sie die reine ostfälische mundart streng durchführte, also vielleicht von einem Ostfalen selbst niedergeschrieben war; keines der hd. elemente nötigt dazu, es schon für die vorlage vorauszusetzen, wenn auch die möglichkeit einer vereinzelten solchen form im princip z.11gestanden werden muss. Es steht also auch von seiten der sprachform nichts im wege, das Tanfgelibnis im ingwäonischen sprachgebiet Ostfalens zulocalisieren.

Zum schluss bleibt uns nun noch die allfassungs\%il unserer sächsischen taufformel zu besprechen. S(herer (s.:31!1) nahm an, dass die uns vorliegende iiberset zung in den anfing:en der Sachsenmission, also bald nach 772 angefortigt soi; als terminus ad quem galt, ihm, wie oben (s.totis) bemerkt und besprochen ist, der erlass der (appitulation de partilus Nis somian. den er (freilich über ein decemimm zu früh: vel. oben s. . 
111 77. mlar 777 ansel\%te. Alle neweren, forscher sind ihm lalin wolulgt: lielle (s. 4:3) nahm sogar 772 als festen termin IIII drol Iormser reichstag dieses jahres als veranlassung an; Inch linewel (s. 4.45) erkliirt, dass die zahl 775 zwar der festen stiit\%umkt، entbehre, aber gewis nicht weit vom richtigen abirre; all .lostes' eiuwände (s. 186), die zu keiner recht klaren pusition gulangen (er schwankt sogar s. 189 zwischen Sachsenmission und l)änemmission, also etwa zwischen 800 und 825), (b)ol er schliesslich (s. 190) entstehung im ersten decennium des 9. jh.'s für das wahrscheinlichste hält, komme ich nachher zutrïck.

Zu praktischer verwendung bei der sächsischen, nach unserer localisierung speciell südostsächsischen mission war die formel zweifellos bestimmt; auch dass sie in die anfänge dieser missionstätigkeit zu setzen ist, kann man, obwol es nicht erweislich ist, als wahrscheinlich gelten lassen. Die frage ist, in welche zeit diese anfänge fielen und wie lange sie etwa gedamert haben werden: die antwort darauf kann uns nur das gesicherte material der kirchengeschichte geben, und wir dürfen hoffen, wenn wir der bewährten führung Haucks vertrauen, zu einem einigermassen gesicherten ergebnis zu gelangen. Ich benutze im folgenden dankbar seine neueste behandlung der christianisierung Sachsens (22,360). Die existenz christlicher kirchen vor Karl dem grossen ist speciell in den suidostgauen Sachsens weder beweisbar noch auch nur wahrscheinlich zu machen. Erst das schwert Karls konnte Sachsen dem christlichen glauben erschliessen, aber erst nach jahrzehntelangen kämpfen. Ich stimme Hauck bei, wenn er (s. 370) ausführt, dass den ersten rache- und plünderungszügen des kaisers ins sächsische gebiet, also den feldzügen der jahre $772,774-775$ und 776 keinerlei missionspläne zur seite giengen: die sicherung der unruhigen grenzen und die erweiterung der fränkischen machtsphäre waren seine ziele. Wenn besonders Einhards annalen das schliessliche resultat der langen fehde, die christianisierung Sachsens, dem kaiser schon als treibendes motiv in seinem ersten zuge von 772 unterschieben, so ist das nichts als eine verwechslung von erfolg und zweck, und zu tiefdringende kenntnis der motive darf man bei der damaligen geschichtschreibung überhaupt nicht erwarten. 
Die tatsache, dass erst im frieden von 776 die religiöse frage zum ersten mal in betrachtung gezogen wird, spricht deutlich genug. Schon damit aber rücken wir von den bisher giltigen datierungen des anfangs der Sachsenmission weg, und es kann jedenfalls von 772 oder 775 keine rede sein. Ein weiterer fehler der betrachtung ist, dass man den begriff des anfangs für unsere datierungsfrage viel zu sehr gepresst hat. Dieser anfang hat in wirklichkeit decennien gedauert, und mehrfach ist das kaum gepflanzte reis des glaubens von dem im innern doch noch heidnisch gesinnten volksstamme mit der wurzel ausgerissen worden. Erst mit der taufe Widukinds 785 konnte eine ruhigere und einigermassen organisierte missionsarbeit wirklich beginnen, und noch im jahre 796 bekennt Alcuin (IIG. Epist. 4, 164): misera Saxonum gens toties baptismi perdidit sacranentum, quia nunquan habuit in corde fidei fundamentum. Der ganze briefwechsel Alcuins ist voll von ausfällen gegen die maledicta generatio und den populus nefandus: es ist daher kaum richtig, wenn Jostes (s. 189) sagt, nach der Capitulatio de partibus hätten sich keine heiden mehr halten können; denn dieser erlass war sicher in seiner strenge gar nicht gänzlich durchführbar, wie denn keine drakonische gewaltmassregel den angestrebten idealzustand zu erzwingen vermag. I)ass den forderungen der Capitulatio nicht nachgelebt wurde, lehren die zwangsverpflanzungen der neunziger jahre, der verlauf des seit 792 aufs hartnäckigste erneuerten kampfes und der umstand, dass Karl in seinem Capitulare saxonicum von 797 die vorschriften seiner älteren Capitulatio aufs neue einschärfte. bei der ansetzung der strafen jedoch bedeutend milder vor\%ugehen sich genötigt fühlte. Man hat demnach das alter unseres Taufgelöbnisses bisher stark überschätzt. und wir werden schwerlich fehlgehen, wenn wir seine ahfassung in die jahre still sich ausbreitender missionstätigkeit, die etwa mit Widukinds taufe anhebt. also in die zeit von 785-792, rund etwa nm 790 setzon.

$7 \mathrm{u}$.Jostes' auseinandersetzungen (s. 186) sei noch rinc bemerkung gestattet. Er behande]t den anch von (1:allio (s. und neuerdings von Wadstein (s. 144) alogedruckten anomymen brief eines deutschen metropoliten an kial den grosen mit

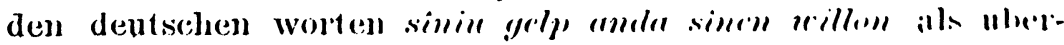


s.1\%umer vin pumpue diaboli, der jetzt als eine dem Amalar ron 'Trive untrigeschobene epistel hinter seinen echten briefen in din .11:. Wpist. 5, $27: 3$ abgedruckt ist (vgl. darüber Hauck :2. 1S1, amm. (i). Iostes set\%t diesen brief um 800-802 an und luingt ihn in bezichung zu Karls Capitula de examinandis Mlusiastiris (Buretius 1, 109). Diese pseudo-amalarische epistel wehört doch aber klärlich, wie auch Hauck annimmt, zu den antwortschreiben auf Karls grosse umfrage über die taufrremonie rom jahre 811 (vgl. Boretius 1, 161. 163. 246. 247; dit: antworten sind, soweit sie erhalten sind, gedruckt in den M(i. Hist. 4, 533. 5, 242); in seinem schreiben an Amalar, dem die iibrigen doch wol ganz oder nahezu gleichlautend, mit dem sie sicher aber inhaltlich identisch waren, fragt der kaiser ausdruicklich betreffs des taufsymbols (ebenda 5, 242), quid sit ejus interpretatio secundum Latinos. Es ist mir unerfindlich, wie Tostes (s. 188, anm. 1) den pseudo-amalarischen brief von dell ïbrigen antworten auf jene umfrage trennen kann mit der begründung, jener beschäftige sich ebenso ausschliesslich mit der taufe von erwachsenen, wie diese mit der kindertaufe; die texte der antworten und Karls schreiben geben für eine solche scheidung, soviel ich sehe, keinen anhalt. Demnach gehört diese Iseudo-amalarische formel weder factisch in dieselbe zeit wie unser Taufgelöbnis, noch ist wegen des verschiedenen wortlauts beider ihr nebeneinanderbestehen zur gleichen zeit unmöglich. Natürlich kamen sie in verschiedenen gegenden zur verwendung; und es gab wol keine allgemeingiltige officielle ïbersetzung des symbolums: jeder missionsbezirk mag seine eigene gehabt haben; das lehrt uns sowol der pseudo-amalarische brief als der eventualzusatz unserer ostfälischen formel.

Bei einer betrachtung des Indiculus superstitionum kann ich mich wesentlich kürzer fassen. Wofür man ihn im ganzen anzusehen hat, scheint mir nicht zweifelhaft. Schwerlich hat Boretius recht, wenn er sagt $(1,222)$ : 'ab homine privato in Saxonia saeculo octavo conceptus esse videtur'; dieser mann müsste geradezu ein verfrühter vorläufer unserer mythologen und folkloristen gewesen sein. Schon Scherer erkannte richtig (s. 318), dass wir es mit einem 'vorläufigen verzeichnis' dessen zu tun haben, 'worauf die königsboten 
oder richtiger die bekehrenden priester zu achten hatten'. Für die auch hier und da (z. b. bei Grimm, Myth. 34, 404), zuletzt von Hauck (2, 393, anm. 2) ausgesprochene ansicht, es handle sich um die capitelüberschriften einer verlorenen abhandlung, kann meines erachtens keinerlei wahrscheinlichkeit angeführt werden, während wir ähnlich gehaltene lakonische programme für synodalberatungen und specielle kaiserliche gesantschaften in die provinzen in menge erhalten haben (vgl. Boretius 1, 62. 65. 99. 102. 114. 121. 122. 138. 140. 149. 161. 162). Der Indiculus kann daher nur eine instruction für königsboten oder eine aufstellung von Capitula de causis cum episcopis et abbatibus tractandis sein. Auf eine volkskundliche und mythologische erläuterung der einzelıen nummern, zu der man die pseudo-augustinische Homilia de sacrilegiis (herausgegeben von Caspari, Christiania 1886) mit glück herangezogen hat, zumal auch sie höchstwahrscheinlich in den nordgegenden des Frankenreichs zu localisieren ist, kann ich hier nicht eingehen: ich verweise dafür ausser auf Haucks bemerkungen $\left(2^{2}, 393\right)$ auf eine germanistischerseits, wie es scheint, gänzlich übersehene abhandlung von Saupe. Her Indiculus superstitionum et paganiarum, ein verzeichnis heidnischer und abergläubischer gebräuche und meinungẹen aus der zeit Karls des grossen, aus zumeist gleichzeitigen schriften erläutert (programm des städtischen realgymnasiums zu Leipzị 1891); sie enthält neben manchem in der beurteilung verfehlten viel brauchbares material aus der zeitgenössischen litejatur. das allerdings aus den capitularien und briefen der karolingischen epoche sich leicht verdoppeln oder verdreifachen liesse. Wichtig ist die spur einer zweiten hs., die den Indirulus enthielt (vgl. Gallée s. 251 und Wadstein s. 14:3): unter auf. zeichnungen, die Franciscus de Nelis, bischof von Antwerpin. sich 1783 aus hss. der klöster Trier, Stablo und Felhternarh anlegte, jetzt in der Briisseler bibliothek aufbewalirt. findell sich Notationes ad indiculum sujerstitionum, hemerkmin 1., 3. und 8. nummer unseres textes, der wätlich ritiert wird. Diese bemerkungen scheinen von Nelis sellos zol stimmen mol erweisen nicht, wie tralles, gemeint hat, did asistell rimes

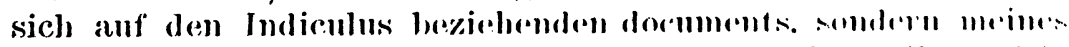

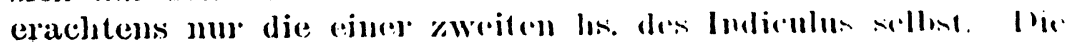


frime wiire riner erncuten nachforschung und untersuchung wol wolt, zumal wir auch über den gesammtcharakter jener anf\%णrbmungen des Nelis nichts wissen.

l'us interessieren vor allem natiirlich die vier deutschen Worte, die ler Indiculus uns ïberliefert. Drei davon sind s:hon lïngst einleuchtend und widerspruchslos gedeutet, für das vierte bisher gänzlich unerklärte lege ich im folgenden 'ine vermutung vor, die wenigstens den versuch einer lösung des darin enthaltenen rätsels macht und einer besseren deutung gern das feld räumen wird. Ueber die dâdsisas $(66,4)$ handeln (irimm in der Myth. ${ }^{4}$ s. 1027 und neuerdings Koegel, Gesch. d. d. lit. 1, 1, 51 (vgl. auch Hauck 22, 761): die ableitung aus dôd und eint'm zum stamme von ahd. sisuua gehörenden worte ist sicher und lässt uns totenzauberlieder darunter verstehen; zur as. form im besondern vgl. Koegel s. 51 . Die nimidas $(66,8)$ hat gleichfalls Grimm (Myth. ${ }^{+}$s. 540. 3, 187) erklärt: der deutliche sinn 'waldheiligtum' nötigt zur anknüpfung an lat. nemus,

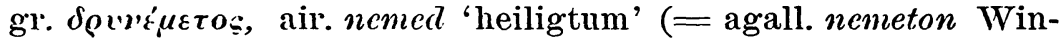
disch, Beitr. 4, 223; vgl. auch Fick, Vergl. wörterb. d. indog. spr. 2 ', 192); es ist der pl. eines diesen auswärtigen verwanten genau entsprechenden nimid. Die bedeutung von nôdfŷr $(66,18)$ ist ausführlich erörtert von Grimm, Myth. ${ }^{4}$ s. 502 und jüngst von Jahn, Die deutschen opfergebr. s. 26: es begegnet schon in den beschlüssen der ersten synode Karlmanns als niedfyr oder neidfyr (Boretius 1, 25), formen, die uns mit ihrem geschlossenen, nach $\hat{\imath}$ hin liegenden $\hat{e}$ (so muss man doch wol die diphthongische schreibung auffassen) an die im afries. ganz gewöhnliche form nêd (Richthofen, Afries. wörterb. s. 945) gemalınen. Ich sehe nicht ein, wie Jostes (s. 190, anm. 1) zu der amnahme kommt, dass in Karlmanns synodalbeschluss ein anderes wort gestanden haben müsse, das der schreiber unseres codex in niedfyr umgeschrieben habe, 'denn weder konnte Karlmann niedfyr schreiben, noch die adressaten es verstehen': der name kam wol den synodalen oder überhaupt der fränkischen kirche zuerst aus einer friesisch sprechenden oder ans friesische angrenzenden gegend zu ohren und wurde sicher verstanden, sonst wäre er hier nicht in den lat. context aufgenommen worden; die annahme einer willkürlichen veränderung durch den schreiber scheint mir gänzlich in der luft zu schweben, 
zumal auch die form im Indiculus durch ihr $\hat{o}$ von der im synodalbeschluss abweicht. - Der bisher unerklärte punkt 24 des Indiculus lautet $(66,27)$ : De pagano cursu, quem yrias nominant, scissis pannis vel calciamentis. Die älteren, sämmtlich lautlich unmöglichen deutungen brauche ich nicht eingehend zu besprechen; sie sind bei Saupe (s. 29) verzeichnet, der eine meines erachtens gleichfalls unmögliche (zu ahd. ero, aisl. jorvi) hinzufügt; auch Koegels anknüpfung an namen wie Iurio und Eurigêr, die selber in ihrem etymon unklar sind (Gesch. d. d. lit. 1, 1, 27, anm. 2), ist wohl abzulehnen. Ebenso ist Massmanns emendation Frias, die noch Müllenhoff als genetiv wie got. gibôs, der aber dann westgerm. erhaltenes $s$ zeigen würde, gelten lassen wollte, lautlich undenkbar wie inhaltlich gewagt und hätte daher von Hauck $(2 \stackrel{2}{3} 393$, anm. 3) lieber nicht erwähnt werden sollen. Ich möchte mit allem vorbehalt folgende deutung zur erwägung stellen. Der zusatz scissis pannis vel calciamentis deutet, wie Koegel richtig gesehen hat, auf einen volksumzug und -brauch, der dem winteraustreiben oder, wie es an andern orten heisst, dem todaustragen verwant ist (vgl. darüber Grimm, Myth. ${ }^{+}$s. 637): der person, welche dabei den winter darstellt, oder der puppe, welche ihn symbolisiert, werden dabei die kleider zerrissen. Diese feier kehrt jährlich wider, sie leitet den schöneren und wärmeren teil des jahres ein, ist gewissermassen die jahresfeier schlechthin. So scheint es mir nicht zu gewagt, in jenem yrias eine ableitung des stammes 'jahr' (as. gî́r, afries. ićr) zu suchen (der pl. scheint, auf cursus bezogen, keine ganz genaue glossierung) und es als 'jahresfest' zu deuten. Lautlich wäre für die stammsilbe von dem as grv, afries. î̀ (phonetisch eigentlich $i \hat{c} r$ ) auszugehen, dessen $\hat{c}$, stark geschlussen und nach $\hat{\imath}$ hin gesprochen, dem hörer und aufzeichner. der kein interesse an allzu genauer reproduction des namens gehabt haben wird, mit dem consonantischen i sich zn ainheitlichem $\hat{\imath}$ (geschrieben $y$, nach ags. weise) verhand. Winthuss des vorhergehenden palatals auf das $\hat{c}$, wie joh iln hior annehme, weist Siebs (\%ur gesch. d. engl.-fries. sir. 1, 2012 und

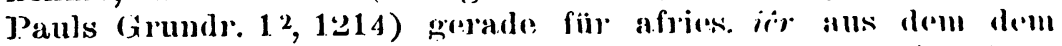
sächsischen benachbarten salerlïndischen nach: ich weiss nicht,

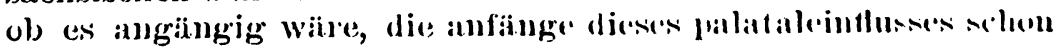


in so frïhe \%(it. \%u set\%en; damn wäre \%ugleich eine möglichliril fiir dic: loculisierung des Judiculus gewonnen. Was die ableitungsille: betrifft, so miisste man wol identität unseres wortes mil affiess. irrich, iêrech, mnd. jầrich annehmen und yrias als *jorisas attfassen; doch scheint mir diese anknüpfung, ubwol sic auch wirklich plualisch genommen einen guten sinn niibe, bei weitem weniger sicher als die etymologisierung der stammsilue.

Bei der gesammtbetrachtung des Indiculus scheint mir seine allzu enge verkoppelung mit dem Taufgelöbnis, die bisher allseitig angenommen worden ist, vom übel gewesen zu sein. Aus der überlieferung beider stücke folgt weder, dass sie in dieselbe zeit gehören, noch auch dass sie aus derselben gegend stammen müssen. Da uns im Indiculus nur vier deutsche worte überliefert sind, kann von einer irgend gesicherten localisierung keine rede sein. Aber wertvoll ist, dass in einem punkte seine sprache sich von der des Taufgelöbnisses unterscheidet: germ. au ist hier durch $\hat{a}$ (dâdsisas), dort durch ô (Saxnôt, gcnôtas, gelôb-) widergegeben. Ijeses $\hat{a}$ erinnert zunächst an das friesische (vgl. Siebs in Pauls Grundr. 1 2, 1231), wohin uns ja auch unsere deutung voll yrias wies, ist aber auch einige mal im Monacensis des Heliand und häufig im Freckenhorster heberegister belegt (Holthausen $\S 29$ und Schlüter bei Dieter s. 97), mit welchem letzteren wir nach dem bistum Münster gelangen würden. Ich möchte die geltung des Indiculus für friesische, den sächsischen benachbarte gaue nach den besprochenen sprachlichen eigenheiten für nicht unwahrscheinlich halten. Noch weniger kann man die abfassungszeit des denkmals genauer fixieren. Nur das ist sicher, dass man sein alter bisher ziemlich stark überschätzt hat. Wenn Koegel (s. 445) von den notizen des Indiculus behauptet, dass sie 'auf grund eines noch ganz ungebrochenen heidentums' zusammengestellt seien, 'wie es im anfange des 9.jh.'s unter den Sachsen gewis nicht mehr zu finden war', so widerspricht das allem, was wir geschichtlich und kirchengeschichtlich von diesen dingen wissen; es zeigt sich hier eine auch sonst in Koegels literaturgeschichte hervortretende bedauerliche unbekanntschaft mit den ergebnissen kirchengeschichtlicher 
SAXONICA. 1. - BURCHARDI, GOT. NAHTAM.

forschung. Unser codex gehört in den anfang des 9. jh.'s: eine datierung des Indiculus um rund 800 wird nicht weit vom richtigen abirren.

JENA, 28. mai $1900 . \quad$ ALBERT LEITZMANN.

\section{NOCH EINMAL GO'TISCH NAHTAM.}

(Zn Beitr. 24, 534 ff.)

Beitr. 24, 534 ff. bemüht sich Pipping, eine erklärung des auffälligen got. dat. pl. nahtam zu geben, der im femininum ohne parallele ist. Was er gegen die gleichsetzung von nahtam mit skr. naktábhis vorbringt, ist alles recht schön und gut: man kann ihm nur darin beistimmen, wenn er gegen die indog. formenriecherei front macht. Auch mir scheint, ehe man eine ganz auffallende germ. form olne weiteres einer ebenso auffallenden indischen gleichsetzt und darin etwas urindog. wittert, sollte man lieber nicht so sehr in die ferne schweifen, sondern sich umsehen, ob das gute nicht näher liegt. Das hat bereits Kahle getan, und Pipping schliesst sich ihm an, in der ansicht nämlich, dass man in nahtam weiter nichts $\mathrm{zu}$ sehen habe, als eine neubildung nach dem masc dat. pl. dagam, die bei der häufigen verbindung dagam jah nahtam entstanden sei, wie das ahd. nahtes nach tages gebildet ist. Aber wozu der lärm? Ausser der ablehnung von .Toh. Schmidts gleichung nahtam $=$ skr. nalitálhis und der deutung von naktálhis als entstanden durch anschluss an aháblis bringt der anfsatz durchaus nichts neues, denn den gedanken, dass nalitam nur analogiebildung nach dagam sei, hat bereits im jalne 1849 oin schriftsteller ausgesprochen, der heute allerdings otwas nnmodern geworden, aber immerhin doch norls ganz lenenswert ist: Jacob Grimm. The urspringliche ahhandlung ershich in der Zs. fda. 7, 45.5 f. und ist wider ahgedrurkt in .I. Cirimms. Kleineren schriften 7, 238 f; dort magr man das niihere nachlesen.

Uebrigens ist anch andern sprachforichern dassello wider.

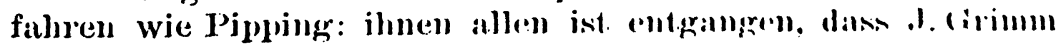

